\title{
Traveling wave solutions for a class of reaction-diffusion system
}

Bingyi Wang ${ }^{1}$ and Yang Zhang ${ }^{1 *}$

\section{"Correspondence:}

zhangyanghit0217@163.com

${ }^{1}$ School of Mathematics Sciences, Harbin Engineering University,

Harbin, 150001, P.R. China

\begin{abstract}
In this paper we investigate the existence of traveling wave for a one-dimensional reaction diffusion system. We show that this system has a unique translation traveling wave solution.
\end{abstract}

Keywords: Reaction-diffusion equation; Traveling wave solution; Existence and uniqueness

\section{Introduction}

This paper deals with the existence and uniqueness of traveling wave solutions of the following reaction-diffusion system:

$$
\left\{\begin{array}{l}
u_{t}=u_{x x}+u\left(1-u-\frac{r v}{1+u}\right), \\
v_{t}=v_{x x}+\frac{b u v}{1+u},
\end{array}\right.
$$

where $r$ and $b$ are positive constant numbers.

In relation to our topic, $\mathrm{Fu}[2,3]$ studied the acid nitrate-ferritin reaction model as follows:

$$
\left\{\begin{array}{l}
u_{t}=\delta u_{x x}-\frac{2 u v}{\beta+u}, \\
v_{t}=v_{x x}+\frac{u v}{\beta+u}
\end{array}\right.
$$

where $\beta$ is a positive constant, $u$ and $v$ represent the concentration of ferritin and acid nitrate, respectively, and $\delta$ is the ratio of diffusion rate. Fu $[2,3]$ showed the existence and uniqueness of traveling solution for the acid nitrate-ferritin reaction model by using the perturbation method. In (1.1) the $U(1-U)$ is the logistic term, which means the birth rate minus death rate of $U$.

There are also many scientists who study the existence, uniqueness, and stability of traveling wave solutions for a reaction-diffusion model in population biology and chemistry. We first recall some existing methods on the existence of traveling waves for the reaction-diffusion model. In [9], Trofimchuk, Pinto, and Trofimchuk studied the traveling wavefronts for a model of the Belousov-Zhabotinskii reaction in a chemical model by

(c) The Author(s) 2021. This article is licensed under a Creative Commons Attribution 4.0 International License, which permits use, sharing, adaptation, distribution and reproduction in any medium or format, as long as you give appropriate credit to the original author(s) and the source, provide a link to the Creative Commons licence, and indicate if changes were made. The images or other third party material in this article are included in the article's Creative Commons licence, unless indicated otherwise in a credit line to the material. If material is not included in the article's Creative Commons licence and your intended use is not permitted by statutory regulation or exceeds the permitted use, you will need to obtain permission directly from the copyright holder. To view a copy of this licence, visit http://creativecommons.org/licenses/by/4.0/. 
constructing upper and lower method solutions. They showed the existence and uniqueness of solution. In [4], Huang investigated the existence of traveling wave for a class of predator-prey systems via the perturbation method. For more details on the existence of traveling wave solutions for other types of diffusion-reaction models, readers can refer to $[5-8,10-13]$ and the references in these papers.

The remaining part of this paper is organized as follows. In Sect. 2, we construct the supersolution and subsolution of (2.1) and introduce some useful lemmas for the main result. In Sect. 3, we show the existence and uniqueness of traveling wave for (2.1).

\section{Preparation}

In this section, we introduce some useful lemmas for the main results of our papers. Note that some reaction-diffusion models in population biology can be rewritten as the form (1.1), and the existence and uniqueness of traveling waves for these reaction-diffusion equations are equivalent to those for (2.1). Throughout this paper, a traveling wave solution of (1.1) always refers to a pair $(U, V, c)$, where $U$ and $V$ are bounded, continuous, nonnegative, and nonconstant functions from $\mathbb{R}$ to $\mathbb{R}$ such that $u(t, x):=U(x-c t), v(t, x):=$ $V(x-c t)$ satisfies (2.1). Clearly, $U(z), V(z)$ satisfy the following wave profile system:

$$
\left\{\begin{array}{l}
U^{\prime \prime}+c U^{\prime}+U\left(1-U-\frac{r V}{1+U}\right)=0 \\
V^{\prime \prime}+c V^{\prime}+\frac{b U V}{1+U}=0 .
\end{array}\right.
$$

There are boundary conditions $(U, V)(-\infty)=(0,1 / r)$ and $(U, V)(+\infty)=(1,0)$. The main purpose of this paper is to study the existence and uniqueness (up to translation) of traveling waves for (2.1).

Next, we construct the supersolution and subsolution of (2.1) that will be used in the following sections. For simplicity, we denote

$$
p(s):=s^{2}-c s+\frac{b}{2}
$$

Due to $c>c^{*}$, where $c^{*}$ is the minimum wave speed (2.1) and equation $p(s)=0$ has two positive roots $\lambda$ and $\lambda+d$, there are

$$
\lambda=\frac{1}{2} c-\sqrt{c^{2}+2 b}, \quad d=\sqrt{c^{2}+2 b} .
$$

In addition, $p(s)<0$, when $s \in(\lambda, \lambda+d)$.

Lemma 2.1 The function $V^{+}(z):=e^{-\lambda z}$ satisfies the equation

$$
\left(V^{+}\right)^{\prime \prime}+c\left(V^{+}\right)^{\prime}+\frac{b}{2} V^{+}=0
$$

for all $z \in \mathbb{R}$.

Proof Since $p(\lambda)=0$, we know

$$
\left(V^{+}\right)^{\prime \prime}+c\left(V^{+}\right)^{\prime}+\frac{b}{2} V^{+}=p(\lambda) V^{+}=0, \quad z \in \mathbb{R} .
$$


Let $0<\gamma<\min \left\{\frac{c}{\delta}, \lambda\right\}$, then $c-\delta \gamma>0, \gamma-\delta<0$. If $z \rightarrow \infty$, then $e^{(\gamma-\delta) z} \rightarrow 0$. There exists $z_{0}>0$ such that

$$
e^{(\gamma-\delta) z} \leq \frac{1}{r} \gamma(c-\delta \gamma), \quad \forall z \geq z_{0}
$$

From the above formula we get

$$
(c-\delta \gamma) \gamma M e^{-\gamma z} \geq r V^{+}(z), \quad \forall z \geq z_{0}
$$

Set $M=e^{\gamma z_{0}}$. Since $\gamma, z_{0}>0$, we know $M>1$.

Lemma 2.2 The function $U^{-}(z):=\max \left\{0,1-M e^{-\gamma z}\right\}$ satisfies the inequality

$$
\left(U^{-}\right)^{\prime \prime}+c\left(U^{-}\right)^{\prime}+U^{-}\left(1-\left(U^{-}\right)-\frac{r V^{+}}{1+U^{-}}\right) \geq 0
$$

for all $z \neq z_{0}$.

Proof When $z<z_{0}$, it can be concluded from the above inequality that $\left(-\infty, z_{0}\right)$ is established on $U^{-} \equiv 0$. When $z>z_{0}$, there is $U^{-}(z)=1-M e^{-\gamma z}$ and $0<U^{-}<1$, so we have

$$
1 \geq \frac{\left(U^{-}\right)}{1+U^{-}}
$$

By calculating (2.2), (2.4), and $M>1$, we know

$$
\left(U^{-}\right)^{\prime \prime}+c\left(U^{-}\right)^{\prime}+U^{-}\left(1-U^{-}\right) \geq r V^{+}(z) \geq \frac{r\left(U^{-}\right) V^{+}}{1+U^{-}}
$$

Thus, we can conclude that (2.3) holds. The proof is completed.

Let $0<\eta<\min \{\gamma, d\}$. Since $0<\eta<\gamma, p(\lambda+\eta)<0$, select

$$
K>\max \left\{M, \frac{-M b}{2 p(\lambda+\eta)}\right\} .
$$

Let $z_{1}=\ln \frac{K}{\eta}, z_{0}=\ln \frac{M}{\gamma}$, and $K>M>1, \eta<\gamma$, therefore $z_{1}>z_{0}>0$.

Lemma 2.3 The function $V^{-}(z):=\max \left\{0, V^{+}(z)-K e^{-(\lambda+\eta) z}\right\}$ satisfies the following inequality:

$$
\left(V^{-}\right)^{\prime \prime}+c\left(V^{-}\right)^{\prime}+\frac{b U^{-} V^{-}}{1+U^{-}} \geq 0
$$

for all $z \neq z_{1}$.

Proof If $z<z_{1}$, from (2.3), we can get that there is $V^{-} \equiv 0$ on $\left(-\infty, z_{1}\right)$. If $z>z_{1}$, there are $V^{-}=V^{+}-K e^{-(\lambda+\eta) z}$ and $U^{-}=1-M e^{-\gamma z}$, and by calculating it is easy to see that

$$
\begin{aligned}
& \left(V^{-}\right)^{\prime}=\left(V^{+}\right)^{\prime}+K(\lambda+\eta) e^{-(\lambda+\eta) z}, \\
& \left(V^{-}\right)^{\prime \prime}=\left(V^{+}\right)^{\prime \prime}-K(\lambda+\eta)^{2} e^{-(\lambda+\eta) z} .
\end{aligned}
$$


Note

$$
\frac{b U^{-}}{1+U^{-}}=b\left[\frac{1}{2}-\frac{M e^{-\gamma z}}{2\left(2-M e^{-\gamma z}\right)}\right] .
$$

We know

$$
\begin{aligned}
\frac{b U^{-} V^{-}}{1+U^{-}} & =b\left[\frac{1}{2}-\frac{M e^{-\gamma z}}{2\left(2-M e^{-\gamma z}\right)}\right]\left(V^{+}-K e^{-(\lambda+\eta) z}\right) \\
& \geq b\left[\frac{1}{2} V^{+}-\frac{1}{2} K e^{-(\lambda+\eta) z}-\frac{M e^{-(\lambda+\gamma) z}}{2\left(2-M e^{-\gamma z}\right)}\right] .
\end{aligned}
$$

Since $\left(V^{+}\right)^{\prime \prime}+c\left(V^{+}\right)^{\prime}+\frac{b}{2} V^{+}=0, K>-M b /(2 p(\lambda+\eta)), 1-M e^{-\gamma z}>0$, and $\gamma>\eta$, we know

$$
\begin{aligned}
\left(V^{-}\right)^{\prime \prime} & +c\left(V^{-}\right)^{\prime}+\frac{b U^{-} V^{-}}{1+U^{-}} \\
\geq & \left(V^{+}\right)^{\prime \prime}-K(\lambda+\eta)^{2} e^{-(\lambda+\eta) z}+c\left(\left(V^{+}\right)^{\prime}+K(\lambda+\eta) e^{-(\lambda+\eta) z}\right) \\
& \quad+b\left[\frac{1}{2} V^{+}-\frac{1}{2} K e^{-(\lambda+\eta) z}-\frac{M e^{-(\lambda+\gamma) z}}{2\left(2-M e^{-\gamma z}\right)}\right] \\
= & -K e^{-(\lambda+\eta) z} p(\lambda+\eta)-\frac{b M e^{-(\lambda+\gamma) z}}{2\left(2-M e^{-\gamma z}\right)} \\
\geq & \frac{b M}{2} e^{-(\lambda+\eta) z}-\frac{b M}{2} e^{-(\lambda+\gamma) z} \\
= & \frac{b M}{2} e^{-(\lambda+\eta) z}\left(1-e^{-(\gamma-\eta) z}\right) \\
\geq & 0 .
\end{aligned}
$$

The proof is completed.

Next, we establish some prior estimates of solutions to nonhomogeneous equations

$$
w^{\prime \prime}(z)+A w^{\prime}(z)+g(z) w(z)=h(z)
$$

We need the following lemmas (Lemma 2.4, Lemma 2.5, Lemma 2.6) (see [3]). For the convenience of readers, we give the details.

Lemma 2.4 Let $A$ be a constant number and $g$ is a continuous function on $[a, b]$. Let $\phi_{1}$ and $\phi_{2}$ be the solution of second-order linear equation $L[y]:=y^{\prime \prime}-A y^{\prime}+g(z) y=0$ on $[a, b]$ such that

$$
\begin{array}{ll}
\phi_{1}(a)=0, & \phi_{1}^{\prime}(a)=1, \\
\phi_{2}(b)=0, & \phi_{2}^{\prime}(b)=1 .
\end{array}
$$

Then we have the following estimates of $\phi_{1}$ and $\phi_{2}$ :

$$
\begin{aligned}
& \left|\phi_{1}(z)\right|+\left|\phi_{1}^{\prime}(z)\right| \leq e^{\left(K_{1}+A+1\right)(b-a)}, \\
& \left|\phi_{2}(z)\right|+\left|\phi_{2}^{\prime}(z)\right| \leq e^{\left(K_{1}+A+1\right)(b-a)}
\end{aligned}
$$

for all $z \in(a, b)$, where $K_{1}=\|g\|_{C[a, b]}$. 
Proof If $g \leq 0$, the Langsky matrix $W\left(\phi_{1}, \phi_{2}\right)$ of $\phi_{1}$ and $\phi_{2}$ can be estimated as

$$
\left|W\left(\phi_{1}, \phi_{2}\right)\right| \geq \frac{1}{A}\left(e^{A(b-a)}-1\right)>0 .
$$

In order to prove (2.6) and (2.7), rewrite the equation $L[y]=0$ as a first-order system

$$
Y^{\prime}=B(z) Y,
$$

where $Y=\left(\begin{array}{c}y \\ y^{\prime}\end{array}\right), B(z)=\left(\begin{array}{cc}0 & 1 \\ -g(z) & A\end{array}\right)$.

Considering $z \in(a, b)$, we know that, for $Y^{\prime}=B(z) Y$ in $Y$, the integral equation is satisfied

$$
Y(z)=Y(a)+\int_{a}^{z} B(\tau) Y(\tau) d \tau
$$

Thus

$$
\|Y(z)\| \leq\|Y(a)\|+\left(K_{1}+A+1\right) \int_{a}^{z}\|Y(\tau)\|
$$

where $\|\cdot\|$ represents the absolute norm, because of $\|B(\cdot)\|=\max \{|g(\cdot)|, A+1\} \leq K_{1}+A+1$, we can easily derive

$$
\|Y(z)\| \leq\|Y(a)\| e^{\left(K_{1}+A+1\right)(z-a)}
$$

Replacing $a$ with $b$ in (2.10), we get

$$
\|Y(z)\| \leq\|Y(b)\| e^{\left(K_{1}+A+1\right)(b-z)}
$$

Now, let $Y=\left(\phi_{1} \phi_{1}^{\prime}\right)^{T},\|Y(z)\|=\left|\phi_{1}(z)\right|+\left|\phi_{1}^{\prime}(z)\right|,\|Y(a)\|=1$, so (2.6) can be obtained from (2.11). Similarly, we let $Y=\left(\phi_{2} \phi_{2}^{\prime}\right)^{T}$ in (2.12) to get (2.7). Note that we prove (2.8), applying the Abel formula and noting $W\left(\phi_{1}, \phi_{2}\right)(b)=-\phi_{1}(b)$, we get

$$
W\left(\phi_{1}, \phi_{2}\right)(b)=-\phi_{1}(b) e^{A(b-z)} .
$$

In order to estimate $\phi_{1}(b)$, we make the second-order equation $\rho(z)=\frac{1}{A}\left(e^{A(z-a)}-1\right)$ on $[a, b]$ have a unique solution $\rho^{\prime \prime}-A \rho^{\prime}=0$. So, there is $\rho(a)=0, \rho^{\prime}(a)=1$. When $z \in(a, b)$, taking note of $\rho \geq 0, g \leq 0$, we find that the function $\psi:=\phi_{1}-\rho$ satisfies

$$
\begin{aligned}
& \psi^{\prime \prime}-A \psi^{\prime}+g \psi=-g \rho \geq 0, \\
& \psi(a)=\psi^{\prime}(a)=0 .
\end{aligned}
$$

According to the maximum principles, we get $\psi \geq 0$, so $\phi_{1} \geq \rho$ on $[a, b]$, then

$$
\phi_{1}(b) \geq \rho(b)=\frac{1}{A}\left(e^{A(b-a)}-1\right)>0 .
$$

Combining (2.13) and (2.14), we know

$$
\left|W\left(\phi_{1}, \phi_{2}\right)(z)\right|=\phi_{1}(b) e^{A(b-z)} \geq \frac{1}{A}\left(e^{A(b-a)}-1\right)>0 .
$$

Therefore, this lemma is proved. 
Lemma 2.5 Let $A$ be a positive constant, and let $g$ and $h$ be continuous functions on $[a, b]$. Let $w \in C([a, b]) \cap C^{2}([a, b])$ satisfy the differential equation

$$
w^{\prime \prime}(z)+A w^{\prime}(z)+g(z) w(z)=h(z), \quad z \in(a, b) .
$$

There is $w(a)=w(b)$. If $-K_{1} \leq g \leq 0,|h| \leq K_{2}$ on $[a, b]$, where $K_{1}, K_{2}$ are constant. There exist a positive constant $K_{1}$ that depends only on the length of $[a, b]$ and a positive constant $K_{3}$ such that $\|w\|_{C[a, b]} \leq K_{2} K_{3}$ holds.

Proof First, we notice that both $w^{\prime}(a+):=\lim _{z \rightarrow a+} w^{\prime}(z), w^{\prime}(b-):=\lim _{z \rightarrow b-} w^{\prime}(z)$ exist, such that $\bar{z} \in(a, b)$ is fixed. Integrating the both sides of $w^{\prime \prime}(z)+A w^{\prime}(z)+g(z) w(z)=h(z)$ from $\bar{z}$ to $z$, we have

$$
w^{\prime}(z)=w^{\prime}(\bar{z})-A(w(z)-w(\bar{z}))-\int_{\bar{z}}^{z} g(\tau) w(\tau) d \tau+\int_{\bar{z}}^{z} h(\tau) d \tau
$$

Since $w, g$, and $h$ are right continuous at point $a$, thus $w^{\prime}(a+)$ exists. Similarly, we also have the existence of $w^{\prime}(b-)$. Let $\phi_{1}$ and $\phi_{2}$ be as shown in Lemma 2.4. For any $z \in(a, b)$, integrating the inequality $w^{\prime \prime}(z) \phi_{1}(z)+A w^{\prime}(z) \phi_{1}(z)+g(z) w(z) \phi_{1}(z)=h(z) \phi_{1}(z)$ from $a$ to $b$, we know

$$
\phi_{1}(z) w^{\prime}(z)-\left(\phi_{1}^{\prime}(z)-A \phi_{1}(z) w(z)+w(a)\right)=\int_{a}^{z} \phi_{1}(\tau) h(\tau) d \tau .
$$

Similarly, we have

$$
-\phi_{2}(z) w^{\prime}(z)+\left(\phi_{2}^{\prime}(z)-A \phi_{2}(z) w(z)+w(b)\right)=\int_{z}^{b} \phi_{2}(\tau) h(\tau) d \tau .
$$

Since $w(a)=w(b)=0$, multiplying (2.15) and (2.16) by $\phi_{1}, \phi_{2}$ respectively, we get

$$
W\left(\phi_{1}, \phi_{2}\right)(z) w(z)=\phi_{2}(z) \int_{a}^{z} \phi_{1}(\tau) h(\tau) d \tau+\phi_{1}(z) \int_{z}^{b} \phi_{2}(\tau) h(\tau) d \tau
$$

Thus

$$
w(z)=\frac{\phi_{2}(z) \int_{a}^{z} \phi_{1}(\tau) h(\tau) d \tau+\phi_{1}(z) \int_{z}^{b} \phi_{2}(\tau) h(\tau) d \tau}{W\left(\phi_{1}, \phi_{2}\right)(z)} .
$$

Since $|h| \leq K_{2}$, this implies

$$
w(z) \leq K_{2} \frac{\phi_{2}(z) \int_{a}^{z} \phi_{1}(\tau) d \tau+\phi_{1}(z) \int_{z}^{b} \phi_{2}(\tau) d \tau}{W\left(\phi_{1}, \phi_{2}\right)(z)} .
$$

Finally, by virtue of (2.6), (2.7), (2.8), and the above inequality, we can get that $\|w\|_{C[a, b]} \leq$ $K_{2} K_{3}$ holds.

Lemma 2.6 Let $A, g$, and $h$ be the same as in the previous lemma. Let $w \in C([a, b]) \cap$ $C^{2}([a, b])$ satisfy $w^{\prime \prime}(z)+A w^{\prime}(z)+g(z) w(z)=h(z)$ in $(a, b)$. If $\|w\|_{C[a, b]} \leq K_{0}$, then there is a 
constant $K_{4}$, only on $A, K_{0}, K_{1}, K_{2}$ and interval length $[a, b]$, so there is

$$
\left\|w^{\prime}\right\|_{C[a, b]} \leq K_{4}
$$

Proof Let $\phi_{1}, \phi_{2}$ be the same as in Lemma 2.4. For any point $z \in(a, b)$, multiplying (2.15), (2.16) by $\phi_{1}^{\prime}$ and $\phi_{2}^{\prime}$, respectively, we have

$$
\begin{gathered}
W\left(\phi_{1}, \phi_{2}\right)(z)\left(w^{\prime}(z)+A w(z)\right)+\phi_{2}^{\prime}(z) w(a)+\phi_{1}^{\prime}(z) w(b) \\
=\phi_{2}^{\prime}(z) \int_{a}^{z} \phi_{1}(\tau) h(\tau) d \tau+\phi_{1}^{\prime}(z) \int_{z}^{b} \phi_{2}(\tau) h(\tau) d \tau .
\end{gathered}
$$

It can be concluded that

$$
w^{\prime}(z)=\frac{\phi_{2}^{\prime}(z)\left[\int_{a}^{z} \phi_{1}(\tau) h(\tau) d \tau-w(a)\right]+\phi_{1}^{\prime}(z)\left[\int_{z}^{b} \phi_{2}(\tau) h(\tau) d \tau-w(b)\right]}{W\left(\phi_{1}, \phi_{2}\right)(z)}-A w(z) .
$$

Under the assumptions of $|h| \leq K_{2}$ and $|w| \leq K_{0}$, we know

$$
w^{\prime}(z) \leq \frac{\left|\phi_{2}^{\prime}(z)\right|\left[K_{2} \int_{a}^{b} \phi_{1}(\tau) h(\tau) d \tau+K_{0}\right]+\left|\phi_{1}^{\prime}(z)\right|\left[K_{2} \int_{a}^{b} \phi_{2}(\tau) h(\tau) d \tau+K_{0}\right]}{\left|W\left(\phi_{1}, \phi_{2}\right)(z)\right|}+A K_{0}
$$

By virtue of (2.6), (2.7), and (2.8), we find that (2.17) holds.

Next, we consider the existence and uniqueness of solution to problem (2.1) within the interval $[-l, l]$. The system is

$$
\begin{cases}U^{\prime \prime}+c U^{\prime}+U\left(1-U-\frac{r V}{1+U}\right)=0, & z \in(-l, l) \\ V^{\prime \prime}+c V^{\prime}+\frac{b U V}{1+U}=0, & z \in(-l, l), \\ (U, V)(-l)=\left(U^{-}(-l), V^{-}(-l)\right), & \\ (U, V)(l)=\left(U^{-}(l), V^{-}(l)\right) . & \end{cases}
$$

By studying some references [1-3], we know that the existence and uniqueness of traveling wave solutions of reaction-diffusion equations in a finite interval can be completed by the following Schauder fixed point theorem.

Lemma 2.7 Let E be a closed convex set in a Banach space, let $T: E \rightarrow E$ be a continuous map such that TE is compact, then $T$ has a fixed point.

Let

$$
\begin{aligned}
& I_{l}:=[-l, l], \quad X:=C\left(I_{l}\right) \times C\left(I_{l}\right) \\
& E:=\left\{(U, V) \in X \mid U^{-} \leq U \leq U^{+} \equiv 1, V^{-} \leq V \leq V^{+}, x \in I_{l}\right\}
\end{aligned}
$$

It is easy to see that $E$ is a closed convex set. In Banach space $X$, we denote the norm $\left\|\left(f_{1}, f_{2}\right)\right\|_{X}=\left\|f_{1}\right\|_{C\left(I_{l}\right)}+\left\|f_{2}\right\|_{C\left(I_{l}\right)}$. Since $U^{-}$and $V^{-}$are nonnegative, we have $U \geq 0, V \geq 0$ for any $(U, V) \in E$. 
Lemma 2.8 Forgiven $\left(U_{0}, V_{0}\right) \in$ E, there is a unique solution $(U, V)$ to the following boundary value problem:

$$
\begin{cases}U^{\prime \prime}+c U^{\prime}+U\left(1-U-\frac{r V_{0}}{1+U}\right)=0, & z \in(-l, l), \\ V^{\prime \prime}+c V^{\prime}+\frac{b U_{0} V_{0}}{1+U_{0}}=0, & z \in(-l, l), \\ (U, V)(l)=\left(U^{-}, V^{-}\right)(l), & (U, V)(-l)=\left(U^{-}, V^{-}\right)(-l) .\end{cases}
$$

In addition, the solution $(U, V)$ satisfies $U>0, V>0$ in $(-l, l)$.

Proof The system is not a coupled system, thus we know that there are existence and uniqueness of $U$ and $V$. By the definition of $U^{-}$and $V^{-}$, we know $U^{-}(-l)=V^{-}(-l)=0$, $U^{-}(l)>0, V^{-}(l)>0$. Since the equation of $V$ is a linear equation, it is easy to see the existence and uniqueness of $V$. Moreover, $V^{\prime \prime}+c V^{\prime} \leq 0, V( \pm l) \geq 0$, by using the maximum principle, there is $V>0$ on $(-l, l)$. Next, we claim the existence and uniqueness of $U$. When $V_{0}$ is a given function, we see that the first equation of (2.19) is second order elliptic equation with boundary condition. Since the term $U\left(1-U-\frac{r V_{0}}{1+U}\right)$ is Lipschitz continuous, according to the argument of regularity of the elliptic problem, the Sobolev imbedding theorem, and the contraction mapping principle, the existence of $U$ is obtained. In addition, by applying the maximum principle, we can see that $U>0, U^{\prime}>0$ in $(-l, l)$.

Now, we define the mapping $T: E \rightarrow X$ by $T\left(U_{0}, V_{0}\right)=(U, V), \forall\left(U_{0}, V_{0}\right) \in E$, where $(U, V)$ is the unique solution to the boundary value problem (2.19). Obviously, any fixed point of $T$ is the solution of problem (2.19).

Lemma 2.9 $T E \subseteq E$.

Proof For given $\left(U_{0}, V_{0}\right) \in E$, denote

$$
(U, V):=T\left(U_{0}, V_{0}\right)
$$

We claim to have $V^{-} \leq V \leq V^{+}$on $I_{l}$. Due to $0 \leq U^{-} \leq U_{0} \leq U^{+} \equiv 1$ and $0 \leq V^{-} \leq V_{0} \leq$ $V^{+}$, we have

$$
\frac{b U^{-} V^{-}}{1+U^{-}} \leq \frac{b U_{0} V_{0}}{1+U_{0}} \leq \frac{b V^{+}}{2} .
$$

Thus

$$
V^{\prime \prime}+c V^{\prime} a+\frac{b U^{-} V^{-}}{1+U^{-}} \leq 0
$$

and

$$
V^{\prime \prime}+c V^{\prime} a+\frac{b V^{+}}{2} \geq 0
$$

for all $z$ in $(-l, l)$. Let $w_{1}=V-V^{-}$, note that $V^{-}=0$ and $V \geq 0$ in $\left[-l, z_{1}\right]$, therefore

$$
w \geq 0, \quad \forall z \in\left[-l, z_{1}\right]
$$


From the third formula of (2.19), we get $w_{1}(l)=0$. In addition, from (2.5) and (2.20), there are $w_{1}^{\prime \prime}(z)+c w_{1}^{\prime}(z) \leq 0$ for all $z \in\left(z_{1}, l\right)$. According to the principle of maximum value, there is $w_{1} \geq 0$ in $\left[z_{1}, l\right]$. And from the two conditions of $w_{1} \geq 0$ and (2.22) in $\left[z_{1}, l\right]$, we can get $V^{-} \leq V$ in $I_{l}$. Similarly, we can conclude that there is $V \leq V^{+}$in $I_{l}$.

Next, we claim that $U^{-} \leq U$ in $I_{l}$. Since $U^{-} \equiv 0$ and $U \geq 0$ on $\left[-l, z_{1}\right]$, thus

$$
U \geq U^{-}, \quad x \in\left[-l, z_{1}\right]
$$

On the interval $\left(z_{0}, l\right]$, we know

$$
U^{\prime \prime}+c U^{\prime}+U\left(1-U-\frac{r V^{+}}{1+U}\right) \leq 0
$$

For simplicity, we denote $\psi(\xi):=\frac{\xi}{1+\xi}$ and $w_{2}:=U-U^{-}$. According to (2.3) and (2.24), we have $w_{2}^{\prime \prime}+c w_{2}^{\prime}+w_{2}\left(1-w_{2}\right)-q(z) w_{2} \leq 0$ on $\left(z_{0}, l\right)$, where

$$
q(z)= \begin{cases}r V^{+}(z) \frac{\psi(U(z))-\psi\left(U^{-}(z)\right)}{U(z)-U^{-}(z)}, & U(z) \neq U^{-}(z) \\ r V^{+}(z) \psi^{\prime}(U(z)), & U(z)=U^{-}(z)\end{cases}
$$

By using of the mean value theorem, we know that $q$ is nonnegative at $\left(z_{0}, l\right)$. In view of (2.23) and (2.19), it is easy to see that $w_{2}\left(z_{0}\right) \geq 0, w_{2} l=0$. Applying the principle of maximum value, we have $w_{2} \geq 0$ on $\left[z_{0}, l\right]$, thus $U \geq U^{-}$on $\left[z_{0}, l\right]$.

Finally, we would like to prove $U \leq U^{+}$on $I_{l}$. Since $U^{*} \equiv 1$ and $V_{0} \geq 0$, we have

$$
\begin{aligned}
& U^{+}( \pm l)=1 \geq U^{-}( \pm l)=U( \pm l) \\
& U^{+^{\prime \prime}}+c{U^{+}}^{\prime}+U^{+}\left(1-U^{+}-\frac{r V^{+}}{1+U^{+}}\right) \leq 0 .
\end{aligned}
$$

Similarly, we find $U \leq U^{+}$on $I_{l}$.

\section{Lemma 2.10 $T$ is a continuous map.}

Proof For $\left(U_{0}, V_{0}\right)$ and $\left(\widetilde{U}_{0}, \widetilde{V}_{0}\right)$ in $E$, it implies

$$
(U, V)=T\left(U_{0}, V_{0}\right), \quad(\widetilde{U}, \widetilde{V})=T\left(\widetilde{U}_{0}, \widetilde{V}_{0}\right)
$$

Let $w_{1}:=U-\widetilde{U}$, it is easy to see that $w_{1}^{\prime \prime}+c w_{1}^{\prime}+w_{1}\left(1-w_{1}\right)+g(z) w_{1}=h_{1}(z)$ and $w_{1}(-l)=$ $w_{1}(l)=0$, where

$$
g(z)= \begin{cases}-r V_{0}(z) \frac{\psi(U(z))-\psi(\widetilde{U}(z))}{U(z)-\widetilde{U}(z)}, & U(z) \neq \widetilde{U}(z), \\ -r V_{0}(z) \psi^{\prime}(U(z)), & U(z)=\widetilde{U}(z),\end{cases}
$$

and

$$
h_{1}(z)=-r \psi(\widetilde{U}(z))\left(V_{0}(z)-\widetilde{V}_{0}(z)\right)
$$


$\psi$ is the same as in Lemma 2.9. Since $0 \leq U, \widetilde{U} \leq 1$, and $0 \leq \xi \leq 1$, we know $0 \leq \psi^{\prime}(\xi) \leq 1$. By applying the mean value theorem, we find $0 \leq \frac{\psi(U(z))-\psi(\widetilde{U}(z))}{U(z)-\widetilde{U}(z)} \leq 1$, when $U(z) \neq \widetilde{U}(z)$.

Note that $\delta>0,0 \leq V_{0} \leq V^{+}, 0 \leq \psi^{\prime}(U(z)) \leq 1$, we have $-K_{1} \leq g \leq 0$, where $K_{1}=$ $r\left\|V^{+}\right\|_{C\left(I_{l}\right)}$. In fact $0 \leq \psi(\widetilde{U}) \leq 1$, it is easy to see $\left|h_{1}\right| \leq r\left\|V_{0}-\widetilde{V}_{0}\right\|_{C\left(I_{l}\right)}$.

By applying Lemma 2.5 , we know that there is a constant $C_{1}$ that depends only on $\delta, c$, $K_{1}, l$ such that

$$
\left\|w_{1}\right\|_{C\left(I_{l}\right)} \leq r C_{1}\left\|V_{0}-\widetilde{V}_{0}\right\|_{C\left(I_{l}\right)}
$$

By the definition of $w_{1}$, we know

$$
\|U-\widetilde{U}\|_{C\left(I_{l}\right)} \leq r C_{1}\left\|V_{0}-\widetilde{V}_{0}\right\|_{C\left(I_{l}\right)}
$$

Let $w_{2}=V-\tilde{V}$ and $\phi(\xi)=\frac{\xi}{1+\xi}$, we have $w_{2}(-l)=w_{2}(l)=0$ and $w_{2}^{\prime \prime}+c w_{2}^{\prime}=h_{2}(z)$, where $h_{2}=-b\left(\phi\left(\widetilde{U}_{0}\right) \widetilde{V}_{0}-\phi\left(U_{0}\right) V_{0}\right)$. This implies

$$
h_{2}=-b\left[\widetilde{V}_{0}\left(\phi\left(\widetilde{U}_{0}\right)-\phi\left(U_{0}\right)\right)+\phi\left(U_{0}\right)\left(\widetilde{V}_{0}-V_{0}\right)\right] .
$$

Since $0 \leq U_{0}, \widetilde{U}_{0} \leq 1$, by using the mean value theorem to

$$
\left|\phi\left(\widetilde{U}_{0}\right)-\phi\left(U_{0}\right)\right| \leq\left|\widetilde{U}_{0}-U_{0}\right|
$$

it implies

$$
\left|\phi\left(\widetilde{U}_{0}\right)-\phi\left(U_{0}\right)\right| \leq\left\|\widetilde{U}_{0}-U_{0}\right\|_{C\left(I_{l}\right)} .
$$

Using

$$
\begin{aligned}
& \left|\widetilde{V}_{0}\right| \leq\left\|V^{+}\right\|_{C\left(I_{l}\right)}, \\
& \left|\phi\left(U_{0}\right)\right| \leq 1
\end{aligned}
$$

and

$$
\left|V_{0}-\widetilde{V}_{0}\right| \leq\left\|V_{0}-\widetilde{V}_{0}\right\|_{C\left(I_{l}\right)},
$$

by (2.27), we infer

$$
\left|h_{2}\right| \leq b\left\|V^{+}\right\|_{C\left(I_{l}\right)}\left\|\widetilde{U}_{0}-U_{0}\right\|_{C\left(I_{l}\right)}+b\left\|V_{0}-\widetilde{V}_{0}\right\|_{C\left(I_{l}\right)} .
$$

Then, from Lemma 2.5 , there is a constant $C_{2}$ depending only on $c$ and $l$ such that

$$
\left\|w_{2}\right\|_{C\left(I_{l}\right)} \leq b C_{2}\left\|V^{+}\right\|_{C\left(I_{l}\right)}\left\|\widetilde{U}_{0}-U_{0}\right\|_{C\left(I_{l}\right)}+b C_{2}\left\|V_{0}-\widetilde{V}_{0}\right\|_{C\left(I_{l}\right)} .
$$

Together with the definition of $w_{2}$, we get

$$
\|V-\widetilde{V}\|_{C\left(I_{l}\right)} \leq b C_{2}\left\|V^{+}\right\|_{C\left(I_{l}\right)}\left\|\widetilde{U}_{0}-U_{0}\right\|_{C\left(I_{l}\right)}+b C_{2}\left\|V_{0}-\widetilde{V}_{0}\right\|_{C\left(I_{l}\right)} .
$$


By virtue of (2.25),(2.26), and (2.28), we have

$$
\begin{aligned}
& \left\|T\left(U_{0}, V_{0}\right)-T\left(\widetilde{U}_{0}, \widetilde{V}_{0}\right)\right\|_{X} \\
& \quad=\|(U, V)-(\widetilde{U}, \widetilde{V})\|_{X} \\
& \quad=\|U-\widetilde{U}\|_{C\left(I_{l}\right)} \\
& \leq b C_{2}\left\|V^{+}\right\|_{C\left(I_{l}\right)}\left\|\widetilde{U}_{0}-U_{0}\right\|_{C\left(I_{l}\right)}+\left(r C_{1} b C_{2}\right)\left\|V_{0}-\widetilde{V}_{0}\right\|_{C\left(I_{l}\right)} \\
& \quad \leq C_{3}\left(\left\|\widetilde{U}_{0}-U_{0}\right\|_{C\left(I_{l}\right)}+\left\|V_{0}-\widetilde{V}_{0}\right\|_{C\left(I_{l}\right)}\right) \\
& \quad \leq C_{3}\left\|\left(U_{0}, V_{0}\right)-\left(\widetilde{U}_{0}, \widetilde{V}_{0}\right)\right\|_{X},
\end{aligned}
$$

where $C_{3}=b C_{2}\left\|V^{+}\right\|_{C\left(I_{l}\right)}+r C_{1}+b C_{2}$. For any given $\epsilon>0$, we set $0<\delta<\frac{\epsilon}{C_{3}}$. By (2.29), for $\varepsilon>0$, there is $\delta>0$ such that

$$
\left\|T\left(U_{0}, V_{0}\right)-T\left(\widetilde{U}_{0}, \widetilde{V}_{0}\right)\right\|_{X}<\epsilon
$$

if $\left\|\left(U_{0}, V_{0}\right)-\left(\widetilde{U}_{0}, \widetilde{V}_{0}\right)\right\|_{X}<\delta$ for any $\left(U_{0}, V_{0}\right),\left(\widetilde{U}_{0}, \widetilde{V}_{0}\right) \in E$. Therefore, $T$ is a continuous map. Thus, the proof is completed.

\section{Lemma 2.11 TE is compact.}

Proof For a sequence $\left\{\left(U_{0, n}, V_{0, n}\right)\right\}_{n \in \mathbb{N}}$ in $E$, let $\left(U_{n}, V_{n}\right)=T\left(U_{0, n}, V_{0, n}\right)$. Because $U^{+}$and $U^{-}$are uniformly bounded on $I_{l}$, and from Lemma 2.6, we know that the sequences $\left\{U_{n}^{\prime}\right\}$ and $\left\{V_{n}^{\prime}\right\}$ are also uniformly bounded on $I_{l}$. Therefore, by applying the Arzela-Ascoli theorem, we obtain that $\left\{\left(U_{n}, V_{n}\right)\right\}$ such that $\left(U_{n j}, V_{n j}\right) \rightarrow(U, V)$ uniformly on $I_{l}$ as $i \rightarrow \infty$. Therefore, $T(E)$ is compact in $E$. So $T$ is precompact.

In view of Lemma 2.8, Lemma 2.9, Lemma 2.10, and Lemma 2.11, we prove that the mapping $T$ satisfies all the assumptions of Lemma 2.7. Therefore, $T$ has a fixed point. This fixed point is the nonnegative solution of system (2.18), so we can get the following result.

Lemma 2.12 System (2.18) has a solution $(U, V)$ on $I_{l}$, and this solution satisfies

$$
0 \leq U^{-} \leq U \leq 1, \quad 0 \leq V^{-} \leq V \leq V^{+}, \quad z \in I_{l} .
$$

\section{The existence of traveling wave solution}

In this section, we would like to show the main result of this paper.

Theorem 3.1 System (2.1) has a unique traveling wave solution $(U, V)$ and

$$
U(\infty)=1, \quad U(-\infty)=0, \quad V(\infty)=0, \quad V(-\infty)=\frac{1}{r} .
$$

Proof Let $\left\{l_{n}\right\}_{n \in \mathbb{N}}$ be an increasing sequence in $\left(z_{1}, \infty\right)$ such that when $n \rightarrow \infty$, there is $l_{n} \rightarrow \infty$, and let $\left(U_{n}, V_{n}\right)$ be the solution of system (2.19) and $l=l_{n}$. And, for any given $N \in \mathbb{N}$, because the function $V^{+}$is bounded on $\left[-l_{N}, l_{N}\right]$, by (2.8), we find that the sequence $\left\{U_{n}\right\}_{n \geq N},\left\{V_{n}\right\}_{n \geq N},\left\{\frac{U_{n} V_{n}}{1+U_{n}}\right\}_{n \geq N}$ is uniformly bounded on $[-l, l]$. Then, by virtue of 
Lemma 2.6, the sequence $\left\{U_{n}^{\prime}\right\}_{n \geq N},\left\{V_{n}^{\prime}\right\}_{n \geq N}$ is uniformly bounded on $[-l, l]$. From (2.19), we have the sequence $\left\{U_{n}^{\prime \prime}\right\}_{n \geq N},\left\{V_{n}^{\prime \prime}\right\}_{n \geq N},\left\{U_{n}^{\prime \prime \prime}\right\}_{n \geq N},\left\{V_{n}^{\prime \prime \prime}\right\}_{n \geq N}$ is uniformly bounded on $[-l, l]$.

Applying the Arzela-Ascoli theorem and the diagonal process, there is a subsequence $\left\{\left(U_{n j}, V_{n j}\right)\right\}$ of $\left\{\left(U_{n}, V_{n}\right)\right\}$ such that

$$
\begin{aligned}
& U_{n j} \rightarrow U, \quad U_{n j}^{\prime} \rightarrow U^{\prime}, \quad U_{n j}^{\prime \prime} \rightarrow U^{\prime \prime}, \\
& V_{n j} \rightarrow V, \quad V_{n j}^{\prime} \rightarrow V^{\prime}, \quad V_{n j}^{\prime \prime} \rightarrow V^{\prime \prime} .
\end{aligned}
$$

When $n \rightarrow \infty, U$ and $V$ in $C^{2}(\mathbb{R})$ are uniformly continuous in a compact set in $\mathbb{R}$. It is easy to conclude that $U^{\prime}>0$ on $\mathbb{R}$. $(U, V)$ is the nonnegative solution of problem (2.1). From the definition of $U^{-}$and $V^{+}$, if $z \rightarrow \infty$, then $U^{-} \rightarrow 1, V^{+} \rightarrow 0$ and

$$
(U, V)(+\infty)=(1,0)
$$

The following proves that $(U, V)(-\infty)=(0,1 / r)$ can be divided into the following steps. First, we prove

$$
\left(U^{\prime}, V^{\prime}\right)(+\infty)=(0,0)
$$

Integrating the both sides of (2.18) from 0 to $z$, we have

$$
\left[U^{\prime}(z)-U^{\prime}(0)\right]+c[U(z)-U(0)]=\int_{0}^{z} U(\tau)\left[U(\tau)-1+\frac{r V(\tau)}{1+U(\tau)}\right] d \tau
$$

Since $U(+\infty)$ exists, we find that $U^{\prime}(\infty)$ exists if and only if the integral

$$
\int_{0}^{\infty} U(\tau)\left[U(\tau)-1+\frac{r V(\tau)}{1+U(\tau)}\right] d \tau
$$

is convergence. Otherwise, it will deviate to $\infty$. And get $U^{\prime}(\infty)=\infty$ from (3.3). Therefore, $U(\infty)=\infty$, which contradicts the existence of $U(\infty)$, so $U^{\prime}(\infty)$ exists. At the same time, we can easily verify $U^{\prime}(\infty)=0$ from $U(+\infty)=1$. Similarly, $V^{\prime}(\infty)=0$ can be obtained by integrating the second expression of system (2.18) from 0 to $z$.

Then, we would like to prove the existence of $(U, V)$ and $1>U(-\infty) \geq 0, V(-\infty) \geq 0$. Since $U$ is increasing, and there is $0 \leq U \leq 1$, thus $U(-\infty)$ exists and $0 \leq U(-\infty) \leq 1$, where $U(-\infty) \neq 1$. If $U(-\infty) \equiv 1$, according to the monotonicity of $U$, then $U \equiv 1$. By (2.18), we have $V \equiv 0$, which contradicts $V \geq V^{-}>0$ in $\left(z_{1}, \infty\right)$. Therefore, $U(-\infty) \neq 1$.

In order to prove the existence of $V(-\infty)$, we need to state that $V \leq 1$ in $\mathbb{R}$. By (1.1), we know

$$
b U^{\prime \prime}+r V^{\prime \prime}+c\left(b U^{\prime}+r V^{\prime}\right)+b U(1-U)=0 .
$$

Integrating the above equality from $z$ to $\infty$, we obtain

$$
\int_{z}^{\infty} b U^{\prime \prime}+r V^{\prime \prime} d \tau+\int_{z}^{\infty} c\left(b U^{\prime}+r V^{\prime}\right) d \tau+\int_{z}^{\infty} b U(1-U) d \tau=0
$$


This implies that

$$
\begin{aligned}
& b U^{\prime}(\infty)+r V^{\prime}(\infty)-\left(b U^{\prime}+r V^{\prime}\right) \\
& \quad+c(b U(\infty)+r V(\infty))-c(b U+r V)+\int_{z}^{\infty} b U(1-U) d \tau=0 .
\end{aligned}
$$

Since $U(\infty)=1, V(\infty)=0, U^{\prime}(\infty)=V^{\prime}(\infty)=0$, we know that

$$
-\left(b U^{\prime}+r V^{\prime}\right)+c b-c(b U+r V)+\int_{z}^{\infty} b U(1-U) d \tau=0
$$

Since

$$
\int_{-\infty}^{\infty} b U(1-U) d \tau
$$

is convergence (by (3.11) and (3.12)), take a constant $K$ such that

$$
K>\int_{-\infty}^{\infty} b U(1-U) d \tau
$$

Let $W=b U+r V-b-K$, since $U \leq 1$ and (2.30), we obtain

$$
W(z) \leq r V \leq r V^{+} \leq r e^{-\lambda z}, \quad \forall z \in \mathbb{R}
$$

Note that $\int_{z}^{\infty} b U(1-U) d \tau \geq 0$, by (3.5), we get

$$
W^{\prime}+c W=\int_{z}^{\infty} b U(1-U) d \tau-K \leq 0
$$

Multiplying the above inequality by the term $e^{c z}$, it is easy to see that $\left[e^{c z} W(z)\right]^{\prime} \leq 0$, which implies that the function $\left[e^{c z} W(z)\right]$ is nonincreasing. Thus, if $-\infty<z_{1}<z<+\infty$, then

$$
e^{c z} W(z) \leq e^{c z_{1}} W\left(z_{1}\right) \leq e^{(c-\lambda) z_{1}} r
$$

Note that $c>\lambda$, if $z_{1} \rightarrow-\infty$, then $W(z) \leq 0$. It is easy to see that $b U+r V-b-K \leq 0$. Thus, we get that $V \leq K / r$ in $\mathbb{R}$.

Next, we prove the existence of $V(-\infty)$ and $V(-\infty) \geq 0$.

Since $V(\infty)=0$ and $V\left(z_{1}+1\right) \geq V^{-}\left(z_{1}+1\right)$, we use the mean value theorem to infer the existence of $\xi_{1} \geq z_{1}+1$ such that $V^{\prime}\left(\xi_{1}\right) \geq 0$. Multiplying the second equation of (3.2) by the term $e^{c z}$, we can easily obtain $\left[e^{c z} V^{\prime}(z)\right]^{\prime}=-e^{c z} \frac{b U V}{1+U} \leq 0$. Thus $e^{c z} V^{\prime}(z)$ is nonincreasing. Since $z>\xi_{1}, e^{c z} V^{\prime}(z) \geq e^{c \xi_{1}} V^{\prime}\left(\xi_{1}\right)>0$, then there is $V^{\prime}<0$ on $\left[\xi_{1}, \infty\right)$. Let $\xi_{2}:=\inf \left\{z \mid V^{\prime}>\right.$ 0 , in $[z, \infty)\}$. Set $\xi_{2}$ be a finite number or $\xi_{2}=-\infty$. If $\xi_{2}=-\infty$, then there is $V^{\prime}>0$ on $\mathbb{R}$. Note that $0 \leq V \leq 1$, we know $V(-\infty)$ exists and $V(-\infty)>0$. If $\xi_{2}$ is a finite number, then there is $V^{\prime}\left(\xi_{2}\right)=0$, which together with the monotonicity of $e^{c z} V^{\prime}(z)$ leads to $e^{c z} V^{\prime}(z) \leq$ $e^{c \xi_{2}} V^{\prime}\left(\xi_{2}\right)=0$, where $z \geq \xi_{2}$. Therefore, there is $V^{\prime} \leq 0$ on $\left(-\infty, \xi_{2}\right]$. We obtain that $V(-\infty)$ exists and $V(-\infty) \geq 0$. Next, we would like to prove

$$
\left(U^{\prime}, V^{\prime}\right)(-\infty)=(0,0)
$$


Under the condition $U(\infty)=1, U^{\prime}(\infty)=0$, integrating the first equation of (2.18) from $z$ to $\infty$, we find

$$
-U^{\prime}(z)+c[1-U(z)]=\int_{z}^{\infty} U(\tau)\left[U(\tau)-1+\frac{r V(\tau)}{1+U(\tau)}\right] d \tau .
$$

By $U \geq 0, U^{\prime} \geq 0$, we have

$$
\int_{z}^{\infty} U(\tau)\left[U(\tau)-1+\frac{r V(\tau)}{1+U(\tau)}\right] d \tau \leq c .
$$

This implies that the improper integral

$$
\int_{-\infty}^{+\infty} U(\tau)\left[1-U(\tau)-\frac{r V(\tau)}{1+U(\tau)}\right] d \tau
$$

converges. Let $z \rightarrow-\infty$, from (3.10) get $U(-\infty)$ exist, we infer that $U^{\prime}(-\infty)$ exists. In addition, because of $U^{\prime} \geq 0$, then $U^{\prime}(-\infty) \geq 0$ is derived. In fact, $U^{\prime}(-\infty)=0$, if $U^{\prime}(-\infty)>$ 0 , then $U(-\infty)=-\infty$, which contradicts the existence of $U(-\infty)$.

Through a similar proof, we can also get $V^{\prime}(-\infty)=0$ and

$$
\int_{-\infty}^{+\infty} \frac{r U(\tau) V(\tau)}{1+U(\tau)} d \tau
$$

Next, we prove that $(U, V)(-\infty)=(0,1 / r)$. Since $U(-\infty), V(-\infty)$ exists, by the improper integrals (3.12), we have

$$
U(-\infty) V(-\infty)=0
$$

Similarly, by virtue of (3.11), we obtain

$$
1-U(-\infty)-\frac{r V(-\infty)}{1+U(-\infty)}=0 .
$$

Since $U(-\infty) \neq 1$, from (3.13) and (3.14), we obtain that $(U, V)(-\infty)=(0,1 / r)$. Therefore, the proof is completed.

\section{Conclusion}

In this paper, we discuss that system (1.1) has a unique translation traveling wave solution by the supersolution and subsolution method and the Schauder fixed point theorem. Moreover, the uniqueness wave solution $(U, V)$ of $(1.1)$ satisfies $U(\infty)=1, U(-\infty)=$ $0, V(\infty)=0, V(-\infty)=1 / r$.

\section{Acknowledgements}

Not applicable.

Funding

Not applicable.

Availability of data and materials

Not applicable. 
Competing interests

The authors declare that they have no competing interests.

\section{Authors' contributions}

This paper is mainly completed by BW and ZY dealt with traveling wave solutions for a class of reaction-diffusion equations. All authors read and approved the final manuscript.

\section{Publisher's Note}

Springer Nature remains neutral with regard to jurisdictional claims in published maps and institutional affiliations.

Received: 30 July 2020 Accepted: 14 March 2021 Published online: 25 March 2021

\section{References}

1. Friedman, A.: Partial Differential Equations of Parabolic Type. Prentice Hall, New York (1964)

2. Fu, S.C.: Travelling waves of a reaction-diffusion model for the acudic nitrate-ferron reaction. Discrete Contin. Dyn. Syst., Ser. B 16, 189-196 (2011)

3. Fu, S.C.: The existence of traveling wave fronts for a reaction-diffusion system modeling the acidic nitrate-ferroin reaction. Q. Appl. Math. 72(4), 649-664 (2014)

4. Huang, W.: Traveling wave solutions for a class of predator-prey systems. J. Dyn. Differ. Equ. 24(3), 633-644 (2012)

5. Joaquin, R.: Existence of traveling wave solutions for a nonlocal reaction-diffusion equation, Dissertations Theses, Gradworks (2007)

6. Li, Z.Y., Wang, M.X., Wu, Y.P., Ye, Q.X.: Traveling wave solutions for reaction-diffusion equations. Nonlinear Anal. 30(6), 3417-3426 (1997)

7. Manjun, M.: Existence of traveling fronts for general reaction systems with nonlinear diffusion. Acta Math. Sci. 1, 113-125 (2012)

8. Riviera, J., Li, Y.: Traveling wave solutions for a nonlocal reaction-diffusion model of influenza a drift. Discrete Contin. Dyn. Syst. 13(1), 157-174 (2010)

9. Trofimchuk, E., Pinto, M., Trofimchuk, S.: Traveling wavefronts for a model of the Belousov-Zhabotinskii reaction. J. Differ. Equ. 254(9), 3690-3714 (2011)

10. Wu, C., Li, M., Weng, P.: Existence and stability of traveling wave fronts for a reaction-diffusion system with spatio-temporal nonlocal effect. Z. Angew. Math. Mech. 9712 (2017)

11. Wu, Y., Zhao, X.: Existence and stability of traveling wave with transition layers for some singular cross-diffusion systems. Phys. D, Nonlinear Phenom. 20, 325-358 (2005)

12. Xin, X.: Existence and stability of traveling waves in periodic media governed by a bistable nonlinearity. J. Dyn. Differ. Equ. 3(4), 541-573 (1991)

13. Ye, Q.X., Li, Z.Y., Wang, M.X., Wu, Y.P. Introduction of Reaction Diffusion Equation. Science Press (2013)

\section{Submit your manuscript to a SpringerOpen ${ }^{\circ}$ journal and benefit from:}

- Convenient online submission

- Rigorous peer review

- Open access: articles freely available online

- High visibility within the field

- Retaining the copyright to your article

Submit your next manuscript at $\gg$ springeropen.com 